\title{
SEMIGROUPS ON FINITELY FLOORED SPACES
}

\author{
BY \\ JOHN D. MCCHAREN
}

\begin{abstract}
This paper is concerned with certain aspects of acyclicity in a compact connected topological semigroup, and applications to the admissibility of certain multiplications on continua. The principal result asserts that if $S$ is a semigroup on a continuum, finitely floored in dimension 2 , then $S=E S E$ implies $S=K$.
\end{abstract}

Introduction. We are concerned here with some aspects of acyclicity in a compact connected semigroup, and applications to the admissibility of a semigroup structure on "finitely floored" spaces (Definition 2). The main result is that if $S$ is a semigroup on a continuum, finitely floored in dimension 2, then $S=E S E$ implies $S=K$. This is a generalization of the result of Cohen and Koch [1], where the same conclusion is obtained assuming that $S$ is a floor for each nonzero $h \in H^{2}(S)$, cd $S \leqq 2$, and $S$ is locally Euclidean except possibly at one point.

A preliminary result which may be of interest in itself is that if $S$ is a semigroup with a zero satisfying $S=E S E$ on a continuum, then for each nonzero $h \in H^{2}(S)$ there exists a pair of idempotents $e$ and $f$ such that $h \mid S e \cup S f \neq 0$.

The notation is that of [4]. In particular, $S$ denotes a topological semigroup, $K$ the minimal ideal, and $E$ the set of idempotents. For a closed set $A$ of $S, S / A$ denotes the space obtained by shrinking $A$ to a point. The cohomology used is that of Alexander-Wallace-Spanier with coefficient group arbitrary. Throughout the paper we shall use reduced groups in dimension 0 . We denote by $A \backslash B$ the complement of $B$ in $A$, the closure of $A$ by $A^{*}$ and the empty set by $\square$. If $\leqq$ is a relation from a space $X$ to a space $Y$, and $y \in Y$, then $L(y)=\{x \in X: x \leqq y\}$; if $B \subset Y$, then $L(B)=\{x \in X: x \leqq b$ for some $b \in B\}$.

The author would like to express his gratitude to Professor R. J. Koch for his many useful suggestions during the preparation of this paper.

Topological preliminaries. If $A$ is a closed subset of a space $X$ and $h$ is an element of $H^{n}(X)$, then $h \mid A$ denotes the image of $h$ under the natural homomorphism $H^{n}(X) \stackrel{i^{*}}{\rightarrow} H^{n}(A)$. The following theorem is due to Wallace [5]:

THEOREM 1. Let $X$ and $Y$ be compact Hausdorff spaces and $\leqq$ a closed relation from $X$ to $Y$ such that $L(A) \cap L(B)$ is connected for each pair of closed subsets $A$ and

Received by the editors June 29, 1970.

AMS 1970 subject classifications. Primary 22A15; Secondary 22A15.

Key words and phrases. Semigroups on continua, acyclicity in compact semigroups, admissibility of multiplication on two-manifolds.

Copyright (ㅇ 1971, American Mathematical Society 
$B$ of $Y$. If $h \in H^{1}(X)$ has the property that $h \mid L(y)=0$ for each $y \in Y$, then $h \mid L(A)=0$ for each closed subset of $Y$.

Definition 2. Let $X$ be a space and $L \neq \square$ a subset of $H^{n}(X)$. A closed subset $F$ of $X$ is called a floor for $L$ if (i) $h \mid F \neq 0$ for each $h \in L$ and (ii) for each proper closed subset $A$ of $F$, there exists an element $h$ in $L$ such that $h \mid A=0$. If $L$ is finite and $F=X, X$ is said to be finitely floored in dimension $n$.

It should be observed that if $X$ is compact and $L \subset H^{n}(X)$ does not contain 0 , then there exists a floor $F$ for $L$. In particular, if $A$ is a closed subset of $X$ such that $h \mid A \neq 0$ for each $h \in L$, then $F$ may be chosen as a subset of $A$.

The following topological lemma is a modified version of that due to Cohen and Koch [1].

LEMMA 3. Let $X$ be a continuum such that $H^{n}(X) \neq 0$, and suppose $X$ is a floor for some subset $L$ of $H^{n}(X)$. If $A$ is a proper retract of $X$, then $H^{n}(X / A) \neq 0$ and $X / A$ is a floor for some subset $L_{0}$ of $H^{n}(X / A)$. Moreover, $L_{0}$ may be chosen to be finite if $L$ is finite.

Proof. Let $\varphi: X \rightarrow X / A$ denote the natural map and $p=\varphi(A) \in X / A$. Consider the commutative diagram in which the top row is exact and $\varphi_{0}^{*}$ is induced by the natural map $\varphi_{0}:(X, A) \rightarrow(X / A, p)$.

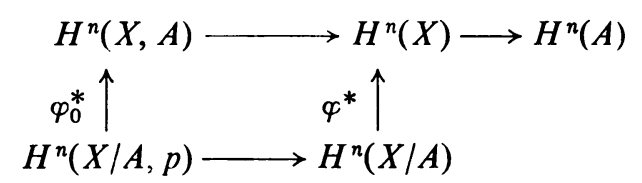

Note that $\varphi_{0}^{*}$ is an isomorphism by the map excision theorem [6]. Let $L_{1} \subset L$ be those elements $h$ in $L$ satisfying $h \mid A=0$. For each $h \in L_{1}$ there exists an element $h_{0}$ of $H^{n}(X / A)$ such that $\varphi^{*}\left(h_{0}\right)=h$. Let $L_{0} \subset H^{n}(X / A)$ be a "section" of $L_{1}$; i.e. for each $h \in L_{1}$ there exists a unique element $h_{0}$ in $L_{0}$ such that $\varphi^{*}\left(h_{0}\right)=h$. Clearly such a set exists and is finite if $L$ is finite. We shall show that $X / A$ is a floor for $L_{0}$.

By the manner in which $h_{0}$ was chosen, it is clear that $0 \notin L_{0}$. Suppose that $B$ is a proper closed subset of $X / A$ and consider the commutative diagram

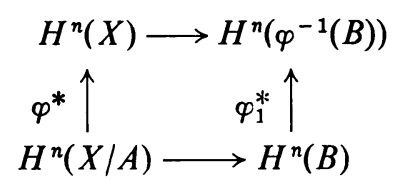

$\varphi_{1}^{*}$ is induced by the restriction of $\varphi$ to $\varphi^{-1}(B)$. Since $X$ is connected, $\varphi^{-1}(B) \cup A$ is a proper closed subset of $X$. Hence there is an element $h$ in $L$ such that $h \mid \varphi^{-1}(B) \cup A$ $=0$. In particular, $h \mid \varphi^{-1}(B)=0$ and $h \mid A=0, h \in L_{1}$, and so there exists an element $h_{0}$ in $L_{0}$ such that $h_{0} \mid B=0$. Our intention is to show that $h_{0} \mid B=0$. Since $h \mid \varphi^{-1}(B)=0$ and $h\left|\varphi^{-1}(B)=\varphi^{*}\left(h_{0}\right)\right| \varphi^{-1}(B)=\varphi_{1}^{*}\left(h_{0} \mid B\right)$, it suffices to show that $\varphi_{1}^{*}$ is injective. 
With this end in mind, two cases are considered:

(i) $p=\varphi(A) \in B$ and

(ii) $p=\varphi(A) \notin B$.

In case (i) $\varphi_{1}$ is a homeomorphism of $\varphi^{-1}(B)$ onto $B$ and thus $\varphi_{1}^{*}$ is an isomorphism. In case (ii) we consider the diagram

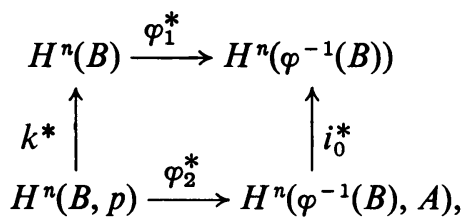

where $\varphi_{2}^{*}$ is induced by the restriction of $\varphi_{0}$ to $\left(\varphi^{-1}(B), A\right)$. Now $k^{*}$ and $\varphi_{2}^{*}$ are isomorphisms by the map excision theorem. It remains to show that $i_{0}^{*}$ is injective. Consider the exact sequence

$$
H^{n-1}\left(\varphi^{-1}(B)\right) \stackrel{m^{*}}{\longrightarrow} H^{n-1}(A) \longrightarrow H^{n}\left(\varphi^{-1}(B), A\right) \stackrel{i_{0}^{*}}{\longrightarrow} H^{n}\left(\varphi^{-1}(B)\right) .
$$

Since $A$ is a retract of $X, m^{*}$ is onto. Therefore $i_{0}^{*}$ is injective. This completes the proof.

Principal results. The following theorem is of use in the sequel and is of independent interest in itself.

THEOREM 4. Let $S$ be a continuum with a zero satisfying $S=E S E$. If $h$ is a nonzero element of $H^{2}(S)$, then there exists a pair of idempotents $e$ and $f$ of $S$ such that $h \mid S e \cup S f \neq 0$.

Proof. Let $F$ be a subset of $E$, minimal relative to (i) being closed and (ii) satisfying $h \mid S F \neq 0$. (Here we are using the hypothesis that $S=S E$.) Since $S$ has a zero, $H^{2}(S e)=0$, so $F$ does not consist of a single element. Thus $F$ may be expressed as the union of two proper closed subsets $A$ and $B$ of $F$. Consider the Mayer-Vietoris sequence

$$
\longrightarrow H^{1}(S A \cap S B) \stackrel{\Delta}{\longrightarrow} H^{2}(S F) \stackrel{J^{*}}{\longrightarrow} H^{2}(S A) \times H^{2}(S B) \longrightarrow .
$$

Because of the minimal conditions of $F, h \mid S A=0$ and $h \mid S B=0$; thus $J^{*}(h \mid S F)=0$. Hence there exists an element $h_{0}$ of $H^{1}(S A \cap S B)$ such that $\Delta\left(h_{0}\right)=h \mid S F$. Define a relation $\leqq$ from $S A \cap S B$ to $A \times B$ as follows: For $x \in S A \cap S B$ and $(e, f) \in A \times B$, let $x \leqq(e, f)$ if and only if $x \in S e \cap S f$. It is easily verified that $\leqq$ is a closed relation from $S A \cap S B$ to $A \times B$. For each subset $C$ of $A \times B, L(C)=\bigcup\{S e \cap S f:(e, f) \in C\}$; therefore $L(C)$ is a left ideal of $S$. Because $S$ has a zero and $S=E S$, it is easily verified that the left ideal $L(M) \cap L(N)$ is connected for each pair of closed subsets $M$ and $N$ of $A \times B$. Now $h_{0}\left|L(A \times B)=h_{0}\right| S A \cap S B=h_{0} \neq 0$, so by Theorem 1 it 
follows that there exists a pair $(e, f) \in A \times B$ such that $h_{0} \mid L((e, f)) \neq 0$. That is to say, $h_{0} \mid S e \cap S f \neq 0$. Consider the commutative diagram

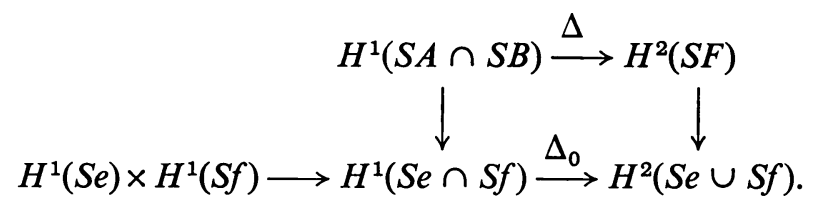

Since $S$ has a zero, $\Delta_{0}$ is injective. Therefore $h \mid S e \cup S f=\Delta_{0}\left(h_{0} \mid S e \cap S f\right) \neq 0$ and the proof is complete.

A point $p$ in a space $X$ is said to be peripheral if there exist small neighborhoods $V$ containing $p$ such that $H^{n}\left(V^{*}, V^{*} \mid V\right)=0$ for all nonnegative $n$. The following lemma follows from Hofmann and Mostert [3, p. 168] and the definition.

LEMmA 5. Let $S$ be a continuum and e an idempotent of $S \backslash K$. If $e \in(S e)^{0}$, then $e$ is peripheral in $S$.

Lemma 6. Suppose that $X$ is a continuum and $X$ is a floor for some subset $L$ of $H^{n}(X)$; then no point of $X$ is peripheral.

Proof. For each neighborhood $V$ of $X$ the natural homomorphism of $H^{n}(X) \stackrel{i^{*}}{\rightarrow} H^{n}(X \backslash V)$ is not injective. Indeed there exists a nonzero element $h$ of $L$ such that $h|X| V=0$. The conclusion follows from the fact that $H^{n}(X, X \backslash V)$ is isomorphic to $H^{n}\left(V^{*}, V^{*} \backslash V\right)$ under the natural homomorphism.

THEOREM 7. Let $S$ be a continuum satisfying $S=E S E$. If $S$ is a floor for some finite subset $L$ of $H^{2}(S)$, then $S=K$.

Proof. Since $S$ is a floor for $L, H^{2}(S) \neq 0$. If $S \neq K$, then $K$ is a proper retract of $S$ [7]. Thus the hypothesis of Lemma 3 is satisfied, and so $S / K$, the Rees quotient modulo $K$, satisfies the hypothesis of the theorem. We may now assume that $S$ has a zero.

By Theorem 4 for each $h \in L$ there exists a pair of idempotents $e_{h}$ and $f_{h}$ in $S$ such that $h \mid S e_{h} \cup S f_{h} \neq 0$. Let $A=\bigcup\left\{S e_{h} \cup S f_{h}: h \in L\right\}$ and observe that $A$ is closed because $L$ is finite. Clearly $h \mid A \neq 0$ for each $h \in L$ and hence $A=S$. We conclude that there exists a finite subset $\left\{e_{i}\right\}_{i=1}^{n}$ of $E$ such that $S=\bigcup_{i=1}^{n} S e_{i}$, and also that $e_{i} \notin S e_{j}$ for $i \neq j$. Then $e_{1} \in\left(S e_{1}\right)^{0}$, so by Lemma $5, e_{1}$ is peripheral in $S$. This establishes a contradiction to Lemma 6 , and the proof is complete.

The class of compact connected 2-manifolds without boundary are covered by the preceding theorem, and other examples are readily constructed. Also, it should be noted that there is an example of a semigroup $S$ with a zero satisfying $S=E S E$ and $H^{2}(S) \neq 0$ [2]. The underlying space of $S$ is a 2-sphere with four closed intervals issuing from a common point $z$ on the 2-sphere. The point $z$ is a zero for $S$ and the other idempotents for $S$ are the free endpoints of the four arcs. In view of Theorems 4 and 7 this example is in a sense a prototype of any such example. 


\section{BIBLIOGRAPHY}

1. H. Cohen and R. J. Koch, Acyclic semigroups and multiplications on two-manifolds, Trans. Amer. Math. Soc. 118 (1965), 420-427. MR 30 \#5283.

2. A. L. Hudson, Example of a nonacyclic continuum semigroup $S$ with zero and $S=E S E$, Proc. Amer. Math. Soc. 14 (1963), 648-653. MR 27 \#1921.

3. K. H. Hofmann and P. S. Mostert, Elements of compact semigroups, Merrill, Columbus, Ohio, 1966. MR 35 \#285.

4. A. D. Wallace, The structure of topological semigroups, Bull. Amer. Math. Soc. 61 (1955), 95-112. MR 16, 796.

5. —, A theorem on acyclicity, Bull. Amer. Math. Soc. 67 (1961), 123-124. MR 23 \#A2196.

6. - The map excision theorem, Duke Math. J. 19 (1952), 177-182. MR 13, 765.

7. — Retractions in semigroups, Pacific J. Math. 7 (1957), 1513-1517. MR 20 \#2400.

\section{Louisiana State University,}

Baton Rouge, Louisiana 70803 